\title{
Acne at ayder referral hospital among patients attending dermatologic clinic, Mekelle, northern Ethiopia, september 2014
}

\author{
Efa Ambaw Bogino, ", Mairie Guizaw Kebede², Alemayehu Bayray Kahsay ${ }^{3}$ \\ ${ }^{1}$ MekelleUniversity, postgraduate in Tropical Dermatology, Norh Ethiopia \\ ${ }^{2}$ MekelleUniversity, Dermatovenereology deparment, North Ethiopia \\ ${ }^{3}$ MekelleUniversity, public health department, North Ethiopia
}

Email address:

ifaasaa@gmail.com(E.A.Bogino),mairguiz@yahoo.com(M.G.Kebede),alemayehub35@gmail.com(A.B.Kahsay)

\section{To cite this article:}

Efa Ambaw Bogino, Mairie Guizaw Kebede, Alemayehu Bayray Kahsay. Acne at Ayder Referral Hospital among Patients Attending Dermatologic Clinic, Mekelle, Northern Ethiopia, September 2014. Science Journal of Clinical Medicine. Vol. 3, No. 6, 2014 , pp. 129-134. doi: $10.11648 /$ j.sjcm.20140306.16

\begin{abstract}
Background: Acne is a chronic inflammatory disease of pilosebceous units with a clinical picture which vary significantly from mild comedonal acne to fulminant systemic disease. Acne has a significant impact on appearance, causing psychosocial and emotional distress, and reducing quality of life of the affected patients. Acne distresses patients due to the fact that it mainly affects the face, being easily visible and constantly present in everyday life. So the aim of this study will be minimizing the gap on magnitude and factors associated with acne at Ayder Referral Hospital.Objectives: To assess the magnitude and factors associated with acne among patients attending dermatologic clinic at Ayder referral hospital, Mekelle, Tigray, Northern Ethiopia.Methods: An institutional based cross sectional study design and sequential convenience sampling technique was implemented at Ayder referral hospital dermatologic Unit until sample size (423) is fulfilled. A total sample size (402) of data was entered and analyzed by SPSS version 16.0. Descriptive and logistic regression analysis was done. Data was presented in the form of tables, graphs and numerical measures. Also it was interpreted by using odds ratio, confidence interval of $95 \%$ and p-value less than 0.05. Result: The magnitude of acne in ARH, dermatologic OPD was 19.4\%. Mean age of patients with acne were 20.6( \pm 4.6$)$ yrs while the mean age at acne start were 17.56( \pm 3.22$)$ yrs. From total of 78 acne patients female accounts 41(52.6\%), Urban resident 66(84\%) and never married were 65(83.3\%). Age (11-20yrs), urban resident, never married individuals, history of acne in the family and cosmetics use were statistically associated with acne on bivariate analysis while family history of acne (AOR7.72(2.99,19.88)CI95\%), cosmetics use (AOR4.83(1.66,14.08),CI95\%) and never married individuals (AOR2.68(1.04,6.91(CI95\%) were significantly associated variables with acne on multivariate analysis.Conclusion and recommendation: The magnitude of acne in ARH was $19.4 \%$ and the associated factors were presence of acne in the family and use of cosmetic make-up. People should avoid use of cosmetics make-up without medical advice.
\end{abstract}

Keywords: Acne, Pilosebaceous Disorder, Ayder Referral Hospital

\section{Introduction}

\subsection{Background}

Acne is a chronic disease of the pilosebceous unit with a clinical picture which vary significantly from mild comedonal acne to the fulminant systemic disease and usually starts in adolescence and frequently resolves by the mid- twenties(1). The peak incidence of acne occurs during adolescence with affecting approximately $85 \%$ of young people between 12 and 24 years of age group but it can also occur during infancy and also about $12 \%$ of women and $3 \%$ of men will continue to have clinical acne until the age of $40 \mathrm{~s}$ (2). The proportion of Acne in hospital based studies in Africa have been reported to be $4.6 \%$ in Ghana(3), $6.7 \%$ in Nigeria(4), $17.5 \%$ in South Africa(5) and $4.5 \%$ between the age 10 and 16 years old in Ethiopia(6)

Acne scars and pigmentation on the face was considered to be a reflection of the severity. Scarring and pigmentation were commonly seen in females than in males $(57.0 \%$ and $48.0 \%$, respectively). The proportion of scar and pigmentation increased with age during adolescence period. 
Psychological impact of acne and stress was related to physical appearance. Adolescent girls were more susceptible than boys to the negative psychological effects of acne $(7,8)$.

Study conducted in USA out of 1013 participants aged 20 years and older showed that, $73.3 \%$ had acne (9). Another study in USA showed that $55 \%$ had some form of acne, $28 \%$ had mild acne, $13 \%$ of which were primarily comedonal. Peaked in the teenage years but aged 21-30yrs (45\%), aged $31-40$ yrs (26\%), and aged 41-50yrs (12\%) had clinical acne (10)

Hospital based study conducted at dermatology OPD in south India showed that 309 patients had acne and the prevalence was $1.068 \%$. Out of the patients $44.3 \%$ were females and $55.7 \%$ were males. The most common age groups were $16-20$ years $(59.8 \%)$. Male patients had more severe acne than female. Mean duration of acne was 45.55 months and the range was 1 month to 25 years. A majority of the patients were college students (33.3\%). Face was involved in all the patients with acne vulgaris. Face alone was involved in $65.4 \%$ followed by the back $28.2 \%$, chest $20.1 \%$, neck $9.4 \%$ and arms $10 \%$. The most common type of lesion was closed comedones and Open comedones were the second most common type of lesion of acne followed by papules, pustules and nodulocysts. From the total patients $60.2 \%$ had mild acne, $30.1 \%$ had moderate acne and $9.7 \%$ had severe acne. Post-acne scarring was seen in $39.5 \%$ of patients. Cheeks were the most common site of post-acne scarring (7).

Community based Study conducted in China showed that no acne was found in subjects less than 10 years of age and only $1.6 \%$ in the 10 -year-old group had acne. The highest prevalence was found in the 19-year-old group (46.8\%). Acne was rare in subjects over 50years of age. It occurred earlier in girls (at 10 years old) than in boys (at12years old). In young teen agers, females showed that higher prevalence than males (11). Another study done in china out of 3163 students 10 to 18 years old showed that prevalence of acne vuglaris was $53.5 \%$ in all adolescents $(51.3 \%$ in males and $58.6 \%$ in females)(12). In other hand the overall magnitude of Acne in Iran, Nigeria and Ethiopia was $85.9 \%$ (13), 90.7\% (14), and $7.7 \%$ (15) respectively

\subsection{Determinant Factors of Acne}

Seborrheic Dermatitis, cigarrate smoking and seasonal variations are most common associated factors with acne. In other hand there are no association between acne development and marker of androgenicity (7).

The use of chocolate and carbonated drinks was the determinant factors associated with the development of acne. Positive sibling's history of acne, frequent use of cosmetic treatment and different types of cosmetics are risk factors for development of acne. Different skin types have different sensitivity to acne which is more common in oily skin than dry and normal skin (16).

Study in Pakistan illustrated that a multivariate logistic regression analysis applied to check the significant combined effect of all study variable on acne showed that skin type (AOR8.81(1.156,8.81), cosmetic used AOR 16.419 (1.181,16.419), positive family history AOR2.76 $(0.813,15.058)$ were significantly associated with acne (17)

A cross sectional study done in Iran shows that being male in gender, younger individuals, increased body mass index and individuals usually subjected to stress are risk factors for acne while frequent wash of faces (more than five times per day) was protective for development of acne (18)

Acne risk is increased with number of family members with acne history. A mother with acne history influenced the severity of acne the most. Increasing pubertal age, seborrhea, the premenstrual phase, mental stress, and sweet and oily foods were recognized as risk factors for development of acne. In contrast, gender, spicy foods, and smoking were not associated with acne. A genetic background is suggested, with mother's acne history being the most important prognostic factor. Skin quality and certain nutrition habits may affect acne (19).

Nutrition habits such as regular eating of sweats, nuts, chocolate and oily foods were associated with acne but no such correlation seen with spicy foods and fasting. In other hand premenstrual phase in females and mental stress were positively associated with acne while smoking did not correlate with acne (20).

Recognizing psychological impact and stress due to the occurrence of acne on the face which is most exposed part of the body. But in our hospitals where many dermatologic cases including Acne were diagnosed and managed there has been paucity of researches to assess magnitude and factors associated with acne. Therefore this study is aimed to assess the magnitude and factors associated with acne at Ayder Referral Hospital, Mekelle, Northern Ethiopia.

\section{Methodology}

\subsection{Study Design and Period}

An institutional based cross sectional study design was implemented at Ayder Referral Hospital from August to September2014.

\subsection{Study Area}

Ayder Referral Hospital, Mekelle, Northern Ethiopia

\subsection{Population}

\subsubsection{Source Population}

All Clients attending dermatologic unit in Ayder Referral Hospital

\subsubsection{Study Population}

All dermatologic patients in Ayder Referral Hospital, Dermatology Clinic during study period

\subsubsection{Inclusion}

All patients with age 11-50 yrs who visit dermatologic unit, Ayder Referral Hospital during study period 


\subsubsection{Exclusion Criteria}

Patients with age 11-50yrs who visit dermatologic unit, Ayder Referral Hospital during study period who is unable to communicate

\subsection{Sample Size Determination and Sampling Technique}

\subsubsection{Sample Size}

The sample size for this particular study was calculated using formula for a single population proportion considering the following assumptions: A 95\% confidence level, margin of error (0.05) and assume maximum variability $(p=0.5)$. These parameters are substituted in the following single population proportion formula.

$$
\frac{\mathrm{n}=(\mathrm{Z} \alpha / 2)^{2} \mathrm{P}(1-\mathrm{P})}{\mathrm{d}^{2}}
$$

Where:

n-the desired sample size

$\mathrm{P}$-Assume maximum variability $(50 \%)$.

$\mathrm{Z} \alpha / 2$-critical value for normal distribution at $95 \%$ confidence level which equals to 1.96 ( $\mathrm{z}$ value $\mathrm{at}=0.05$ )

d-The margin of error taken as 0.05

Therefore, the formula yields 384 sample size and adding $10 \%$ (39clients) for non-respondent the total minimum larger sample size was 423 .

\subsubsection{Sampling Technique}

A sequential convenience sampling method was implemented. All clients attending dermatologic units that were eligible for sample were interviewed and examined for presence of Acne during data collection period.

\subsection{Study Variables}

\subsubsection{Dependent Variables} Acne

\subsubsection{Independent Variables}

Socio demographic characters: Age, Sex, Marital status, residence, occupation, educational status, and religion.

Other factors; smoking, alcohol consumption, cosmetic make-up use, family history of acne

\subsection{Data Management and Analysis}

The collected data was checked for completeness and logical sequence of responses. The completed questionnaires were checked for completeness, consistency and were coded by the principal investigator. Data was entered in to SPSS version 16.0 software package for analysis. Data was presented by tables, graphs and numerical numbers. Descriptive statistics was computed to determine the magnitude of Acne. Bivariate analysis was carried out between dependent and independent Variables to determine the differential of magnitude of Acne by independent variables separately. Binary logistic regression analysis after checking model fitness was made to obtain odds ratio and the confidence interval of statistical associations. Variables with p-value less than 0.25 were taken to multivariate analysis. The strength of statistical association was measured by adjusted odds ratios and $95 \%$ confidence intervals. The significance was declared at $\mathrm{P}<0.05$.

\subsection{Quality Control}

To ensure data quality, questionnaire was translated from English to local language by translators and back to English to compare the consistency. Data collectors were trained for one day. Every day at the end of the data collection discussion was made with the data collectors; problems encountered find the solution. The completeness of the questionnaire was checked before data entry. Since no similar facilities which manage dermatologic cases around study area pretesting was done at dermatology clinic of Ayder referral hospital prior to actual data collection. Participants in this pretest were not included in the study. The principal investigator was checked collected data for completeness and corrective measures were taken accordingly.

\subsection{Ethical Consideration}

Ethical clearance was obtained from the ethical review committee of the CHS of Mekelle University and ethical clearance was taken. Accordingly, permission letter was secured from medical directors at Ayder Referral Hospital. In additional of the study participants were informed about purpose of the study and oral/verbal consent was obtained before interview, examine and also ensure during each step of data collection. Respondents were notified that they have the right to refuse or terminate at any point of the interview and the information provided by each respondent was kept confidential. Patient identification variables such as name, house number, phone number were not used in the study. This study did not inflict harm on or expose the patients to un necessary risk.

\section{Result}

\subsection{Socio-Demographic Characteristics}

A total of 402 patients participated in the study making the response rate of $95.2 \%$. The mean $( \pm \mathrm{SD})$ age of respondents was $24.4( \pm 8.6)$ years. The most common age groups of respondents were 16-20yrs (139/34.6\%). Fifty five percent of respondents were female and $45 \%$ were males while $382(95 \%)$ of the respondents were orthodox by religion. Only $36(9 \%)$ of the respondents were illiterate while others complete their primary, secondary and above twelve135 $(33.6 \%), 140(34.8 \%)$ and $91(22.6 \%)$ respectively. Students constitute the largest occupational status (37.8\%) followed by self-business oriented individuals $(20.6 \%)$. Nearly threefourth $(74.4 \%)$ of respondents were living in urban and about $61.4 \%$ of them were never married.

Out of 402 respondents $78(19.4 \%)$ of them have clinical characteristics of Acne. The majority of acne occurs in the age group of $16-20$ (47cases/60.3\%) which is $26.9 \%$ in male and $33.3 \%$ in females of the same age group. The mean $( \pm \mathrm{SD})$ 
age of individuals with acne was $20.6( \pm 4.6) y r s$ and the mean age $( \pm$ SD) at acne initially start was $17.56( \pm 3.22)$ yrs. Out of acne cases observed female accounts 41(52.6\%), males 37 (47.4) and orthodox religion was $71(91 \%) .52 .6 \%$ (41) of the individuals with acne were students and they complete secondary school by their educational level. Urban resident accounts $66(84.6 \%)$ while $65(83.3 \%)$ of them were never married. The mean duration of acne lesion was 32.7 months with a range of two months to 14 years.

\subsection{Clinical Characteristics of Acne}

Out of 78 patients with acne comedone appear in 71(91\%) with involvement of face and multiple sites (face, chest, back) $45(57.7 \%)$ and $26(33.3 \%)$ respectively. Papulopustular in $60(76.9 \%)$ with involvement of face and multiple sites $36(46.2 \%)$ and $22(28.2 \%)$ respectively. Nodules appear only in $10(12.8 \%)$ of the patients. $70(89.7 \%)$ of acne patients have greasy face and acne vulgaris were the most common $(75(96.2 \%))$ acne variant observed. On over the entire most involved site with acne was face which accounts $98.7 \%$ while the rest was chest and back.

Two-third of the acne vulgaris were moderate type of acne while mild and severe type accounts $21(26.7 \%)$ and $3(3.8 \%)$ respectively. Patients' on follow up accounts 18 out of 21 mild cases, while 38 out of 52 moderate cases were newly diagnosed cases. $19(24.4 \%)$ of individuals with acne develops atrophic form of acne scar and 32(41\%) were develop post inflammatory hyperpigmentation. 64(82.1\%) of patients with acne were no complain of itching and only $12(15.4 \%)$ were recurrent. $15(19.2 \%)$ were flare during menstruation while 33(42.3\%) were flare during stress and only two patients complaining of aggravation of acne lesion with sweaty foods. Most (76(97.4\%) of patients who has acne had no any history of medication before appearance of acne. $61(78.2 \%)$ of acne patients were newly diagnosed while $17(21.8)$ of the patients were on tretnoin and doxycycline treatment.

$29(7.2 \%)$ of the respondents have history of acne in the family of which 28 of them were in the first degree relatives. Out of the respondents 9 and 43 individuals had habit of smoking and alcoholic respectively. $35(8.7 \%)$ of the patients have habit of using cosmetics, out of these $16(4 \%)$ of them were used steroid containing while $19(4.7 \%)$ were used oily emollients.

\subsection{Multivariate Analysis}

Multivariate analysis was employed to assess the net effect of selected variables. The result of multiple logistic regression models revealed that person with history of acne in the family, an individual who uses cosmetics and never married individuals were significantly associated with development of acne. Person who has acne in family was7.72 times more likely to develop acne (AOR7.72 $(2.99,19.88)$ CI95\%) than who have no acne in family, Person who use cosmetic make-up were 4.83 more likely to develop acne (AOR4.83(1.66,14.08)CI95\%) than others who did not use cosmetics while individuals who were never married was $2.68(1.04,6.91) \mathrm{CI} 95 \%)$ times more likely to develop acne than individuals who were married (table1).

Table 1. Multivariateanalysis on acne and its associated factors at ARH, Dermatologic OPD, and September2014.

\begin{tabular}{|c|c|c|c|c|c|}
\hline \multirow{2}{*}{ Variables } & \multirow{2}{*}{ Category } & \multicolumn{2}{|c|}{ Acne } & \multirow{2}{*}{$\operatorname{COR}(95 \% \mathrm{CI})$} & \multirow{2}{*}{$\operatorname{AOR}(95 \% \mathrm{CI})$} \\
\hline & & Yes & No & & \\
\hline \multirow{3}{*}{ Age } & $11-20 y r s$ & 51 & 130 & $5.73(2.2,14.9)$ & $2.07(0.54,7.93)$ \\
\hline & $21-30 y r s$ & 22 & 121 & $2.65(0.96,7.31)$ & $0.78(0.22,2.72)$ \\
\hline & $\geq 30 \mathrm{yrs}$ & 5 & 73 & 1 & 1 \\
\hline \multirow{3}{*}{ Educational status } & Illiterate & 3 & 33 & $0.34(.09,1.25)$ & $0.72(0.13,3.99)$ \\
\hline & Primary & 15 & 120 & $0.44(0.21,0.94)$ & $0.44(0.17,1.17)$ \\
\hline & Secondary & 41 & 99 & $1.61(0.86,2.99)$ & $1.28(0.57,2.85)$ \\
\hline \multirow{3}{*}{ Occupation } & Above 12 & 19 & 72 & 1 & 1 \\
\hline & Student & 41 & 111 & $2.93(1.51,5.67)$ & $1.08(0.44,2.66)$ \\
\hline & Employed & 16 & 78 & $1.63(0.75,3.53)$ & $2.99(0.86,10.35)$ \\
\hline \multirow{4}{*}{ Place of residence } & Un employed & 7 & 24 & $2.31(0.84,6.34)$ & $0.75(0.30,1.87)$ \\
\hline & Self business & 14 & 111 & 1 & 1 \\
\hline & Urban & 66 & 233 & $2.15(1.1,4.16)$ & $1.3(0.59,2.88)$ \\
\hline & Rural & 12 & 91 & 1 & 1 \\
\hline \multirow{2}{*}{ Marital status } & Never married & 65 & 182 & $3.90(2.07,7.39)$ & $2.68(1.04,6.91) *$ \\
\hline & Married & 13 & 142 & 1 & \\
\hline \multirow{2}{*}{ Alcohol history } & Yes & 13 & 30 & $1.96(0.97,3.96)$ & $2.22(0.91,5.40)$ \\
\hline & No & 65 & 294 & 1 & 1 \\
\hline \multirow{2}{*}{ Acne in family } & Yes & 19 & 10 & $10.11(4.4,22.8)$ & $7.72(2.99,19.88) *$ \\
\hline & No & 59 & 314 & 1 & 1 \\
\hline \multirow[t]{2}{*}{ Cosmetic use } & Yes & 20 & 15 & $7.1(3.44,14.68)$ & $4.83(1.66,14.08) *$ \\
\hline & No & 58 & 309 & 1 & 1 \\
\hline
\end{tabular}

COR: Crude odds ratio, AOR: Adjusted odds ratio, * significantly associated variables at $\mathrm{p}<0.05$ 


\section{Discussion}

Magnitude of acne in ARH dermatologic OPD was $19.4 \%$.The most common affected age groups were between $16-20 \mathrm{yrs}(60.3 \%)$ with mean age of $20.6( \pm 4.6)$. The mean age at which acne initially start was $17.56(3.22)$. The mean duration of acne was 32.7 months with a range of two months to $14 y$ rs. Acne vulgaris was most common acne variants which accounts $96.2 \%$. Presence of acne in the family, use of cosmetic make-up and unmarried were significantly associated variables in multivariate analysis.

Magnitude of acne varies worldwide from $90.7 \%$ at Nigeria (14), 85.9\% at Iran (13), 34.7\% at Syria (18), $7.7 \%$ at Ankober Ethiopia (15), 4.7\% at Taiwan (10), Also in this study the magnitude of acne was $19.4 \%$ which was higher than Taiwan and Ankober Ethiopian study but it was lower than Syria, Iran and Nigeria study. The possible reason could be for lower prevalence of the research was done at the community level. Even if the study design was similar for the higher prevalence and this research; while the study area and study units were different which was school and hospital based respectively.

The mean age of acne occurrence in this study was around eighteen years (17.56) which was almost similar to research showed those 20yrs at USA (9), 18yrs at Syria and Iran and the highest prevalence was seen between 16-20yrs age group $(34.6 \%)$ which was almost similar to the study done in china which showed highest prevalence between 15-19 age groups.

Regarding to the prevalence of acne by sex; it was found that $52.6 \%$ were females in this study which was also similar in different studies done at USA (10), Iran (19) and Nigeria (14). Study in the Ghana (3) shows that prevalence of acne is more common in the urban than rural population which was similar to this study (84.6\% were urban residents) but another study in Nigeria (14) indicated that acne prevalence was almost similar in urban and rural. The possible reason could be due to variation in socio-demographic character and life styles of the community

According to study in India (7), Nigeria (14), and China(13) mild(60/56) form of acne was more common than moderate(30/24) and severe(10/21) but in this study the moderate $(66.7 \%)$ form of acne was more common than mild and severe. The possible reason might be delay in early visiting of health institution, low awareness on the diagnosis and treatment of the disease and people might not give attention for the disease during mild stage. Comedone is the most common (91\%) type of lesion and face is the mainly affected site by acne according to study done in south western India which is also similar to this study. Hospital based cross sectional study in India showed that acne flare during stress accounts $45 \%$ which is almost similar with this study which accounts $42 \%$.

There had been different factors which were significantly associated with acne. Use of medically unadvisable cosmetic make-ups which may have steroid containing and oily emollients or comedogenic products in their ingredients was some of the risk for development of acne. In this study using of cosmetic make-up was significantly associated with acne with AOR of 4.83(1.66,14. 08(CI95\%) which is similar to the study done in China (10) and Pakistan (17).

The presence of acne in the family was one of the variables which was significantly associated with acne with AOR of 7.72(2.99,19.88(CI95\%) which was also suggested by study done in Pakistan (17)and India (16)

Nutrition habits such as regular eating of sweats, nuts, chocolate and oily foods and premenstrual phase in females and mental stress were positively associated with acne in study done in Iran (20) but in this study there is no correlation with this variables.

Smoking cigarette was significantly associated with acne as stated by USA study and also drinking alcohol was significantly associated with acne which was stated in study done in China. But this both variables have no association in this study.

\section{Conclusion}

The magnitude of acne in the Ayder Referral Hospital dermatologic OPD was $19.4 \%$.

Presence of acne in the family, history of cosmetics use and never married individuals were common risk factors for the development of acne.

\section{Recommendation}

For health professionals

- The clinicians should focus on persons who have positive family history of acne regarding early detection and treatment and also further study should be done on genetic predisposition of acne

For the community leaders

- Community leaders should mobilize and educate the people to avoid use of cosmetic make-up without medical advice.

\section{Acknowledgement}

I would like to thank dermatovenereology department and mekelle university for giving me the opportunity to do thesis. My deepest gratitude also goes to data collectors and respondents without whom this thesis would not have enrealized. Lastly my special thanks pass to my beloved friends for their constructive comments.

\section{Referances}

[1] Tony Burns, Stephen Breathnach, Neil Cox,etal. Rook's Textbook of Dermatology, Vol.3rd, 7thed. Blackwell Publishing Ltd (UK); 2004: 2137-50.

[2] Peter C, Vande K, Joost S. Papulo squamous and Eczematous Dermatoses. In: Jean L, Joseph L, Ronald P(eds). Bologna Dermatology. USA: MosbyElsevier,2008:115-134.

[3] Doe PT, Asiedu A, Acheampong JW.etal. Skin disease in Ghana and UK. Int Dermatol2001; 40: 323-326. 
[4] Yahya H. Change in pattern of skin disease in Kaduna, North central Nigeria. Int Dermatol2007; 46: 936-943.

[5] Hart Shorne ST. Dermatological disorder in Johannesberg, South Africa. Chin ExpDermatol2003; 28:611-665.

[6] Figueria JI, Fuller LC, Abraha A, etal. The prevalence of skin disease among school children in rural Ethiopia. A preliminary assessment of dermatologic needs. Peadiatr Dermatol1996; $13: 378-381$.

[7] Balaji A, Devinder M. Profile of acne vulgaris. Ahospitalbased study from South India. Indian JDermatol Venereol Leprol,2009;75:272-8.

[8] Chikeung Y, Lynnhwee Y, Leihong X etal.A Communitybased Epidemiological Study of Acne Vulgaris in Hong Kong Adolescents. Act a DermVenereol2002;82:104-107.

[9] Roderick J, Nicole E. Hywel C. et al. Global Burden of Skin Disease. Journal of Investigative Dermatology (2014) 134, 1527-1534.

[10] Perkins A, Maglione J, Hillebrand G, etal. Acne vulgaris in women: prevalence across the life span. J Womens Health, 2012; 21(2): 223-30

[11] Yiwei S, Tinglin W, Cheng Z, et al. Prevalence of Acne Vulgaris in Chinese Adolescents and Adults. ActaDermVenereol, 2012; 92: 40-44

[12] $\mathrm{Wu} \mathrm{T}$, Mei S, Zhang J, etal. Prevalence and risk factors of facial acne vulgaris among Chinese adolescents. Int J Adolesc Med Health, 2007; 19(4): 407-12.

[13] Yi-Chien Y, Hung-Pin T, Chien-Hui H. Female Gender and Acne Disease Are Jointly and Independently Associated with the Risk of Major Depression and Suicide: A National Population-Based Study.BioMed Research International; 2014, 1:7.

[14] Husain Y,Acne vulgaris in Nigerian adolescents prevalence, severity, beliefs, perceptions, and practices.International Journal of Dermatology; 2009, 48:498-505

[15] ShimelisNigusseDoni, Vitalia,Murgia, Mateo Beraldo, Dagnachew Shibeshi. A Mobile Community Dermatologic Clinic in AnkoberWoreda, Central Ethiopia. Community Dermatology Journal 2013; 8: 1-12.

[16] Haritha, MadhaviK. Acne and Quality of Life. A Study from Tertiary Care Centre in South India.IOSR Journal of Dental and Medical Sciences;2013,6(3):59-62

[17] Shazia M, Mohammed A, Mubashra A, etal. Precipitating factors of acne vulgaris in females. Ann. Pak. Inst. Med.Sci.2009;5(2):104-1-7.

[18] Waqar A, Nik Nairan A, Sabzali M, etal. Socio demographic Characteristics of Acne among University Students in Damascus, Syria. Epidemiology Research International, 2014; $10: 4$

[19] Mohammad T, Bahareh M, Mohammad N. Prevalence of acne and its impact on the quality of life in highschool-aged adolescents in Yazd, Iran. Journal of Pakistan Association of Dermatologists2013;23(2):168-172.

[20] Zahra S, Helmut O and Christos Z. Prevalence, Severity, and Severity Risk Factors of Acne in High School Pupils: A Community- Based Study. Journal of Investigative Dermatology (2009)129,2136-2141 